\title{
LA MEDICIÓN DE TELEVISIÓN EN ESPAÑA: ESTADO DEL ARTE Y PROPUESTA PARA EL CÁLCULO DE LA AUDIENCIA HÍBRIDA TOTAL
}

\author{
THE MEASUREMENT OF TELEVISION IN SPAIN: STATE OF ART AND \\ PROPOSAL FOR THE CALCULATION OF THE TOTAL HYBRID AUDIENCE
}

Jose Antonio Cortés Quesada y Teresa Barceló Ugarte

| joseacortes.20@gmail.com | tbarcelo@ceu.es |

Universidad San Pablo CEU

Resumen: El entorno televisivo ha sufrido una auténtica revolución digital en los últimos años, amparada por una amplia oferta de soportes y plataformas, y la facilidad de conexión e intercambio de contenidos audiovisuales. Este cambio ha fomentado la transformación del consumo televisivo hacia una hiperfragmentación de la audiencia, digna de estudio. En este contexto, la principal empresa encargada de la medición de audiencia de la televisión lineal en España, Kantar Media, ha decidido dar un paso más allá hacia una medición crossmedia; lo que significa aplicar los métodos de mensuración tradicionales con tratamientos digitales. Pero el gran proceso de digitalización empuja a realizar una medición de audiencia mucho más completa de la que se está planteando en el sector audiovisual; de esta manera, en la presente investigación se muestra, como alternativa, una medición híbrida total para los contenidos audiovisuales que se emiten en las diferentes plataformas. Y se señala, al mismo tiempo, la necesidad de la creación de un organismo que vele por la veracidad de los datos que se puedan obtener, y por la privacidad y derechos de los usuarios. Palabras clave: audiencia; audímetro; medición; hiperfragmentación; medición crossmedia; medición híbrida. 


\begin{abstract}
The television environment has suffered an authentic digital revolution in recent years, covered by a wide range of media and platforms, and the ease of connection and exchange of audiovisual content between them. This change has encouraged television consumption has also evolved towards hyper-fragmentation of the audience, worthy of study. In this context, the main company in charge of audience measurement of linear television in Spain has decided to go a step further towards a cross-media measurement; using traditional methods, changing them to digital ones. But the great process of digitization pushes us to make a measurement of the audience much more complete than what is being considered in the audiovisual sector; In this way, in the present investigation, a total hybrid measurement of the audiovisual contents that are issued in the different platforms is proposed. Noting, at the same time, the need for the creation of an organization that ensures the veracity of the data that can be obtained, and for the privacy and rights of the users. Keywords: Audience; Audiometer; Measurement; Hyper-fragmentation; Cross-media Measurement; Hybrid Measurement.
\end{abstract}

\title{
1. Introducción y contextualización
}

Nos encontramos ante una oportunidad única para realizar un estudio de los espectadores que consumen contenidos audiovisuales. Amparados por el avance tecnológico y la conectividad, se puede hablar de una audiencia hiperconectada (Quintas y González, 2018). A partir de aquí, es posible obtener diversos datos sobre los usuarios que, desde los diferentes soportes que ofrecen contenidos audiovisuales, realizan un consumo muy diferente al que tenía lugar apenas cinco años atrás.

El usuario ha cambiado de rol; ha dejado atrás ese comportamiento pasivo en el que esperaba a que la televisión tradicional programase su consumo televisivo. Ahora es él mismo el que se encarga de decidir qué quiere ver, dónde y cuándo. Es por esto por lo que se habla de user generator flow y producer controller flow. Los usuarios pueden incluso llegar a crear grandes comunidades en torno a un contenido audiovisual, con la innegable ayuda de las redes sociales (Marinelli y Giandomenico, 2014).

Se han ido dando pequeños pasos en el sector audiovisual por la evolución tecnológica y digital, que han sido mucho más rápidas de lo que nadie hubiese podido imaginar. Todos estos cambios han dado lugar a una gran fragmentación de la audiencia. Los espectadores tienen ante sí una amplia oferta de canales televisivos de donde escoger contenidos. Además, aparte de esta multiplicidad de canales, se ha ampliado el número de soportes y sistemas que ofrecen diferentes contenidos y formas de consumirlos (Vaca, 2009). 
Otra circunstancia reseñable es la existencia de las redes sociales y la posibilidad de interactuar entre diferentes usuarios de cualquier parte del mundo. Esto ha dado lugar a algo inimaginable, la utilización de estas redes sociales para interactuar al visualizar cualquier contenido que se emita en televisión, ya sea en directo o diferido, un fenómeno conocido como televisión social. Algunas redes sociales han destacado más que otras a la hora de interactuar en torno a un contenido audiovisual, como, por ejemplo, Twitter (Quintas y Neira, 2018).

Al mismo tiempo, aunque se produce de manera leve, existe un descenso de la presencia de televisores, DVDs y Blu Ray en los hogares, pero, por el contrario, hay un ascenso de SmartTV, teléfonos móviles inteligentes (smartphones) y tabletas (Barredo, 2017).

Ante este panorama, surgen dudas sobre si el sistema de medición de audiencias sigue siendo tan efectivo como lo fue antaño, ya que, en el caso de España, en veinte años tanto el sistema de visionado como las cadenas existentes y los canales, la tecnología y la conectividad han evolucionado de manera considerable y el audímetro no lo ha hecho al mismo nivel (PuroMarketing, 2018).

Algunos cambios que sí se han producido en la medición de audiencia de la televisión lineal ${ }^{1}$ en España han estado protagonizados por la empresa encargada de este cometido, Kantar Media, que optó, a partir de 2015, por fusionarse con ComScore, encargada de la medición en medios digitales (Dircomfidencial, 2016). Esta unión planteó nuevos retos en la medición, así como en el resto de los soportes y sistemas desde donde se pueden consumir contenidos audiovisuales.

Del mismo modo, se ha producido una proliferación de plataformas que ofrecen contenidos audiovisuales como es el caso de Netflix, HBO, Amazon Prime o Hulu y de proveedores de televisión por suscripción como, por ejemplo, Movistar +, que ya cuentan con más de siete millones de usuarios en España (Barlovento, 2019).

Estos cambios en el sector han llevado a que en los últimos tiempos se hayan producido fusiones comerciales por parte de empresas competidoras para participar en el nuevo ecosistema audiovisual. Encontramos casos como la unión de servicios de contenidos en streaming: Netflix y Movistar +, Vodafone con $\mathrm{HBO}$, o el de los tres principales grupos audiovisuales de televisión en España, para crear un sistema que permita mejorar la experiencia de

[01] Se puede definir televisión lineal como la televisión que se consume al mismo tiempo que se emiten sus contenidos. No es necesario que éstos sean en directo, también puede tratarse de contenido en diferido. 
visualización de consumos audiovisuales por parte del espectador, LOVEStv (Barlovento, 2019).

Teniendo en cuenta estos factores, es necesario cuestionar si la medición que se está utilizando es la correcta o si, aprovechando la cantidad de actores involucrados que conviven en este nuevo sector audiovisual, podría ofrecerse una mejora de mayor envergadura, que permita la obtención de datos complejos, completos y lo más verídicos posibles, acordes con la situación actual.

\section{Objetivos y metodología}

El objetivo general que se pretende alcanzar con esta investigación es revisar el estado de las audiencias en la actualidad.

Por otra parte, hay otros objetivos específicos a los que se pretende llegar:

$\rightarrow 1$. Analizar los cambios que están produciendo las nuevas tecnologías en la manera de consumir televisión y cómo la interactividad que proporcionan estas herramientas provoca una convergencia entre los contenidos audiovisuales y los usuarios que los consumen. Creando cada vez más una necesidad de protagonismo por parte del usuario consumidor.

$\rightarrow 2$. Proponer la medición híbrida total en la televisión lineal como una alternativa a la medición de audiencia actual. Y aprovechar, de esta manera, los diferentes softwares y sistemas que se están desarrollando para controlar el consumo del contenido en Internet, los implantados en la televisión conectada, las mejoras de los audímetros, así como las aplicaciones de medición que están surgiendo en los últimos tiempos.

En esta investigación se van a tener en cuenta los consumos de contenidos audiovisuales actuales y pasados. Es decir, la televisión lineal y los múltiples formatos que permiten al usuario elegir qué ver y dónde.

Al mismo tiempo, el enfoque de la investigación que se plantea es el de demostrar, mediante un panel de expertos y una revisión bibliográfica ${ }^{2}$ de la historia del audímetro, la televisión y la inversión publicitaria en este mercado, que los datos que se podrían obtener de los consumidores de la televisión

[02] En esta revisión se tuvo en cuenta todo el material bibliográfico que se encontró sobre la historia del audímetro, así como su evolución. Al mismo tiempo, se tuvieron en cuenta las investigaciones y artículos cuya temática giraba en torno al cambio en la medición de la audiencia en la televisión lineal, así como los nuevos métodos que algunas empresas, de manera individual, estaban llevando a cabo. Así, se podía tener una visión de la situación muy amplia, lo que permitió un análisis de la evolución de la audimetría más profundo. 
lineal se están desperdiciando y es necesaria una evolución en la recogida de estos. Así, se van a describir los diferentes modelos televisivos que existen en la actualidad, para entender la necesidad del cambio en la medición de audiencias televisivas.

En cuanto a los expertos consultados, debían ser aquellos vinculados con los protagonistas de la medición de audiencia de la televisión lineal en la actualidad. Los cuestionarios y entrevistas se realizaron entre diciembre de 2018 y marzo de 2019. Entre ellos se encuentran:

$\rightarrow$ Eduard Nafría, director de Insights y Desarrollo de Negocio de Kantar Media, empresa encargada en España de obtener datos cuantitativos y cualitativos de los espectadores que consume un determinado contenido televisivo.

$\rightarrow$ Miguel Ángel Fontán, director Regional Sur de Europa de Kantar Media.

$\rightarrow$ Ricardo Vaca, CEO de Barlovento Comunicación S. L, empresa responsable de consultar y analizar los datos emitidos por Kantar Media.

$\rightarrow$ Ignacio Gómez Hernández, director de Análisis y Nuevos Proyectos de RTVE.

$\rightarrow$ Pere Vila, director de Tecnología, Innovación y Sistemas de RTVE.

$\rightarrow$ Andrés Díaz Díaz, subdirector de Ingeniería y Mantenimiento de Sistemas de TV en Mediaset España.

$\rightarrow$ José Girón, responsable de los Sistemas de Tecnología (CIO) de Atresmedia.

$\rightarrow$ Alejandro Perales Albert, asesor Técnico de la Asociación de Usuarios de la Comunicación.

$\rightarrow$ Begoña Gómez, directora Técnica de la Asociación Española de Anunciantes. 
$\rightarrow$ Francisco Asensi, experto en innovación en contenidos, negocios digitales y nuevos modelos de entretenimiento digital, OTT $^{3}$ y vídeo.

$\rightarrow$ Vicente García, cofundador de FLUZO, empresa responsable de un nuevo método de medición de audiencia a partir de una app.

A la hora de plantear la medición de la audiencia, se tuvo en cuenta si su uso estaba siendo el correcto o no. El medio televisivo, ya que es el medio audiovisual más consumido de todos, es el que además presenta unas tasas de implantación superior entre los hogares españoles (Quintas y Neira, 2018). Además, es el medio que mayor inversión publicitaria ha recibido en 2018, aunque haya sufrido un estancamiento en los últimos años (Barlovento, 2018), y el que más influenciado ha estado por las audiencias (Madinaveitia y Mapi, 2015).

Finalmente, se ha elegido España para la realización de este trabajo debido a que existe una escasez de investigaciones respecto a la mejora o cambio de la medición de audiencia de la televisión lineal en este país, por lo que aumenta el estímulo de indagar en la cuestión ${ }^{4}$. Además, España es considerado como uno de los países con un buen mercado audiovisual (Quintas y Neira, 2018). Aparte de tener una de las mejores audiencias sociales por la gran aceptación de los dispositivos móviles en la sociedad (Santiago e Ignacio, 2015).

\section{El futuro de la medición de audiencia en la televisión lineal}

El mundo audiovisual está viviendo un cambio radical producido por la introducción de las nuevas tecnologías y la forma de consumir contenido por parte de los usuarios.

El comienzo de esta etapa tiene su origen con la popularización de Internet (2006) $)^{5}$. Los espectadores interactúan con los contenidos, incluso los comparten, y pueden llevarlos a convertirse en un auténtico éxito con su interacción. Para que este escenario sea posible, es necesario que el equipamiento en los hogares sea el óptimo. En España, según el informe de 2018 del Instituto Nacional de Estadística (INE), más del 98 por ciento de los hogares

[03] Servicios Over The Top. Son aquellos servicios que se ofrecen a través de Internet. Conocidos como servicios de libre transmisión.

[04] La última tesis doctoral en España que abordaba la medición de audiencias de la televisión lineal fue defendida en 1993 por Amparo Huertas Bailén: Sistemas de medición de audiencia en televisión: Evolución y Tendencia. No tenemos en cuenta la tesis doctoral llevada a cabo por Kevin Arnaldo Mejía, El uso de las redes sociales para la medición de audiencias: el modelo de Honduras como una propuesta de paradigma global, dado que centra su investigación en Honduras.

[05] Fue en este año cuando el número de usuarios aumento a 2.000 millones en todo el mundo. 
disponían de teléfono móvil y casi el 86,4 por ciento ${ }^{6}$ tenía acceso a Internet. En el gráfico 1, se observa cómo la televisión es el dispositivo que reina en casi todos los hogares españoles ( 99,1 por ciento), aunque se aprecia una leve bajada de la misma y una subida notable de otros aparatos que favorecen un consumo audiovisual diferente al disfrutado hasta ahora.

llustración 1. Equipamiento tecnológico de los hogares españoles (2017-2018).

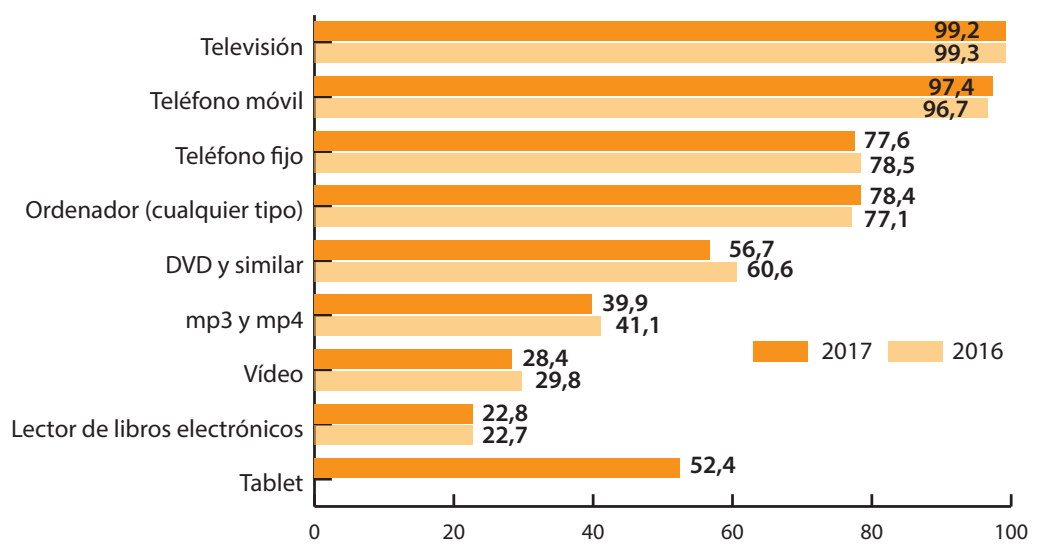

Fuente: INE (Instituto Nacional de Estadística).

Según las profesoras, Ana González y Natalia Quintana (2016), hay un número escaso de autores que analicen el panorama audiovisual actual y cómo afecta éste a la medición de audiencias.

Existen unas excelentes condiciones tecnológicas que favorecen una audiencia hiperconectada. Los medios tradiciones se adaptan a esta nueva situación poco a poco y algunos como la televisión «viven la transición digital como un complejo proceso de innovación continua y disruptiva» (Freire, 2015: 445).

Durante varias décadas, el audímetro ha sido el dispositivo estrella para obtener los datos relativos a la audiencia televisiva; o lo que es lo mismo, la cantidad de espectadores de la televisión lineal. Con el avance de la tecnología y la manera en la que cambian los consumos audiovisuales en la actualidad, muchos son los que ven el audímetro ${ }^{7}$ como algo completamente obsoleto.

[06] Un 4,6 por ciento más que los datos reflejados en el informe correspondiente a 2015.

[07] El audímetro es el aparato que, acoplado al receptor de radio o televisión, sirve para medir las horas concretas en que están encendidos y el tiempo total de funcionamiento, con el fin de determinar el índice de audiencia. Es decir, es el dispositivo encargado de recoger la información de una cantidad determinada de hogares, para informar del número de personas y características de las mismas, que han consumido un contenido audiovisual en concreto. La metodología usada en la medición de la audiencia televisiva es, mayoritaria- 
Incluso recelan de su invariabilidad pese a los avances tecnológicos (PuroMarketing, 2018).

En 2018 se produjo uno de los hitos más significativos de esta apuesta por el cambio, cuando la revista Variety publicó que la cadena de televisión estadounidense CBS estaba pensando no renovar su contrato con la gran empresa medidora de audiencias, Nielsen. Esto podría suponer un gran revés para el mundo de la medición de audiencias tradicionales, si se tiene en cuenta que CBS es la cadena líder en televisión lineal en Estados Unidos y el contrato con Nielsen asciende a la astronómica cifra de 100 millones de dólares anuales (PuroMarketing, 2018).

Arancha Lázaro, ejecutiva de cuentas de la empresa Kantar Media en España, afirmaba para el periódico El País que «a las cadenas pequeñas les afecta más cuando deja de verles un panelista ${ }^{8}$. Las cadenas de televisión grandes tienen datos de audiencias más estables» (Hidalgo, 2018). Por lo tanto, las cadenas autonómicas siempre serán las más afectadas en el caso de los datos de audiencia.

Una de las mejoras que propone la empresa Kantar Media es la creación de lo que se conoce como el botón de invitado, botón que se añadió en 2018 al mando del audímetro para tener en cuenta a los invitados que consumían contenido audiovisual en casas ajenas a las suyas. Esta decisión de mejorar e innovar también levantó polémica, ya que se afirmaba que, si se implementaba, se produciría un aumento del consumo mensual de televisión entre el 6 por ciento y el 8 por ciento, perjudicando la fiabilidad de los datos (La Vanguardia, 2017).

En este punto, es necesario hacer una explicación acerca del guest-shift (botón de invitado) y el time-shif:

$\rightarrow$ Time-Shift: a la medición obtenida a través de los paneles tradicionales de Kantar Media hay que sumarle el consumo mediante los decodificadores, que permiten ver el contenido audiovisual recuperado; es decir ver un programa o serie después de su emisión. Se conoce como

mente, cuantitativa, ya que el fin de esta medición es la de conocer la cantidad de personas que están viendo un contenido audiovisual determinado. Si bien es cierto que, en esta medición, existe una parte cualitativa, aunque no ha tenido una importancia tan relevante como la cuantitativa. Los miembros de los hogares que poseen un audímetro tienen, a su vez, un papel activo en el correcto funcionamiento de estos dispositivos, ya que necesitan aportar información al aparato a través del mando a distancia. Para cada persona que conforma un hogar, el audímetro es capaz de almacenar cuánto tiempo consume una determinada cadena, cuánto tiempo no ha accedido al televisor, así como la cantidad de minutos que sí que lo consume y el cambio de cadena. En España, en 2019, hay 4.875 audímetros.

[08] Hogar que posee un audímetro. 
Time-Shift a la suma de la audiencia tradicional de las personas que consumen un producto audiovisual durante los siete días posteriores a su emisión original.

$\rightarrow$ Guest-Shift: botón en el mando a distancia del audímetro que cuenta a las personas no residentes de un hogar pero que sí están consumiendo un contenido en el mismo. Por ejemplo, familiares que vienen de visita, invitados para una ocasión determinada - como un evento deportivo-- o hijos que no habitan en la casa de sus padres; es el caso de los padres divorciados o trabajadores/as del hogar. De esta manera se incrementaría un 5 por ciento los datos de audiencias (Fernández, 2017).

Para Carlos Lamas, exdirector adjunto de AIMC, el principal inconveniente del audímetro es que en casi setenta años de existencia sólo ha tenido una gran mejora tecnológica en toda su historia. Se pasó del enfoque de audímetro de aparato o set-meter (se controlaban los aparatos de televisión y se proporcionaba la audiencia en términos de hogares) a los actuales audímetros de botones o people-meters, donde además se intenta medir el número y características de los televidentes (Lamas, 2010).

Entre las voces más destacadas en el tema se puede encontrar a Vicente García, cofundador de la empresa FLUZO, encargada de proporcionar nueva tecnología a la medición de audiencia en televisión, radio y medios online. Este experto afirma que es necesario estar atentos a las nuevas tecnologías y no perder de vista a las nuevas generaciones, que son las que más se desmarcan de la medición tradicional y serán los consumidores del futuro. «Las generaciones que están consumiendo de esta manera no son el target comercial propiamente dicho, pero esa generación es la más interesante de estudiar, porque serán los futuros consumidores; como la industria no los tenga en cuenta y no se modernice, va a tener lugar una situación realmente complicada para el sector» (García, 2018).

En los últimos tiempos se han ido presentando nuevas formas de medir la audiencia en la televisión lineal, pero es cierto que muchas de ellas han quedado en el olvido o se ha demostrado finalmente que los datos que ofrecían no se acercaban a la realidad. Un ejemplo de ello fue la medición de audiencia a través de las redes sociales.

En la actualidad los contenidos audiovisuales que se emiten en televisión se consumen acompañados de las nuevas tecnologías. Además, «en el nuevo ecosistema televisivo no sólo están presentes las cadenas, sino que también 
se han introducido con fuerza las empresas de telecomunicación (Vodafone, Movistar+, etc.), los fabricantes de dispositivos (Samsung, Sony, Nexus) y los operadores OTT, como Hulu o Netflix» (Quintas y Neira, 2018).

A la hora de explicar la relación entre la medición de audiencias y las redes sociales, es necesario tener en cuenta el concepto de multitasking: la habilidad de realizar diferentes tareas al mismo tiempo. En el mundo de la televisión se puede entender este concepto como la capacidad de consumir un contenido audiovisual mientras que el espectador interactúa con otro dispositivo. También se puede hablar del término multipantalla (Prompy, Helena, Jimenez, José y Cox, 2014).

Esta forma de consumir cualquier contenido audiovisual y, al mismo tiempo, interactuar en redes sociales dio lugar al concepto conocido como audiencia social. «Los espectadores, en su doble rol de espectador y usuario, encuentran en las herramientas sociales un canal directo para manifestar la pertenencia activa con el programa emitido» (Claes y Deltell, 2015).

En España, Kantar Media compró The Data Republic, empresa encargada de monitorizar y analizar datos en tiempo real. Esta compañía desarrolló Tuitele, capaz de analizar las conversaciones en las redes sociales y, por ende, con la ayuda de Kantar Media, medir la audiencia social a través de Twitter. La empresa encargada de medir y analizar los datos de audiencia de la televisión lineal también se hizo con los derechos de Fisheye Analytics; una startup ${ }^{9}$ capaz de realizar un seguimiento de los medios sociales existentes. Por su parte, Carlos Sánchez, cofundador de Tuitele, afirmó antes de la compra por parte de Kantar Media que «el gran valor de la audiencia social es que complementa a los datos de audimetría» (Zenith, 2013).

La manera en la que funciona Tuitele permite, como declaró Sánchez, integrar nuevas capas de información a los datos ofrecidos por $\operatorname{Kantar}^{10}$ :

$\rightarrow$ Autores únicos: el número de personas que tuitean sobre un contenido televisivo en concreto.

$\rightarrow$ Audiencia única: la cantidad de gente total que ha visto un tuit.

$\rightarrow$ Impresiones: la cantidad de veces que un tuit ha sido servido y, de esta manera, ha sido visto.

[09] Empresa que presenta unas altas posibilidades de crecimiento y pertenecer a un modelo de negocio cambiable (Dorantes, 2018).

[10] Tras la compra de Tuitele por parte de Kantar Media, ésta ofreció unas nuevas métricas de audiencia. 
$\rightarrow$ El número de tuits y retuits publicados durante la emisión; también se tienen en cuenta los publicados antes y después de la misma.

$\rightarrow$ La cantidad de tuits publicados por minuto: al mismo tiempo es capaz de calcular el mayor volumen de tuits totales que están relacionados con una emisión.

Se pudo comprobar tras esta compra, cómo los espacios televisivos comenzaron a integrar hashtags ${ }^{11}$ para que los espectadores pudieran interactuar a través de Twitter y así participar en esa nueva medición de audiencia. Por otro lado, «la medición de esta audiencia en tiempo real aporta información cuantitativa y cualitativa muy valiosa para las cadenas de televisión» (Quintas y Neira, 2014).

Sonia Got, Social Media Manager de Mediaset España, y trabajadora en la productora Plural en 2012, declaró que los datos y la función de Tuitele no era desbancar al audímetro, sino que era necesario unificar ambos sistemas. Además, después de anunciar la compra por parte de Kantar Media, se compararon los datos ofrecidos por ambas empresas. Se usó como ejemplo el programa Hay una cosa que te quiero decir, de Mediaset España que, según Kantar, obtuvo un 19,6 por ciento de share, mientras que Tuitele publicó que el 24 por ciento de usuarios de Twitter se decantaban por El señor de los anillos, que emitió La Sexta, con más de 50.000 tuits procedentes de 31.000 usuarios diferentes (Barrio, 2012). Se puede apreciar cómo los datos de audiencia ofrecidos y la información que aporta la medición de Tuitele no son los mismos, aunque se pueden compaginar para tener una información mucho más completa sobre el comportamiento de la audiencia.

Según un informe publicado por Barlovento Comunicación, en octubre de 2018 se registraron un total de 17,5 millones de tuits sobre emisiones televisivas, siendo Telecinco la cadena más tuiteada, seguida de Televisión Española y La Sexta. En cuanto al género de la emisión, un 45,8 por ciento de los tuits van destinados a programas de entretenimiento, mientras que un 39,8 por ciento son para los concursos y un 8,5 por ciento para los programas relacionados con la información.

Sánchez afirmó que lo interesante de la información de Tuitele no era la información cuantitativa que podían ofrecer sus mediciones, sino la cualitativa. Se puede saber la opinión de los espectadores, los gustos, qué no les

[11] Cualquier palabra, frase o expresión precedida del carácter almohadilla (\#) que se usa en las redes sociales para crear pequeñas comunidades que hablan o interactúan sobre un mismo tema. 
está gustando del producto que consumen durante la emisión del mismo. Además, no dudó en afirmar que este tipo de redes sociales fomentaban el consumo de las emisiones en directo. «La televisión se convierte en una experiencia más amplia, una experiencia que además queremos hacer no en diferido sino en directo, cuando el programa se está emitiendo. La televisión social hace que los espectadores quieran consumir más televisión en directo» (Regidor, 2013).

\section{Diferentes propuestas para la mejora o cambio en la medición de audiencia en la televisión lineal}

En 2016 se confirmó que el acuerdo ${ }^{12}$ firmado un año antes por Kantar y ComScore no había avanzado tanto, ya que no se terminaba de desarrollar un buen panel de medición (Dircomfidencial, 2016).

Al conferir tanta importancia a esta nueva medición crossmedia, no se tiene en cuenta la medición de la audiencia en la televisión lineal, que sigue anclada y apenas ha sufrido ligeras modificaciones desde el nacimiento del audímetro. Es de justicia señalar que, cuando el audímetro nació, era el método más fiable. Además de ser más rápido que, por ejemplo, las encuestas.

Según un artículo publicado por Carlos Lamas para la Asociación para la Investigación de Medios de Comunicación (AIMC), no se ve lejos la llegada de un medidor multimedia que permita analizar los datos de cada usuario de una manera eficaz y rápida. Anima incluso a asumir los elevados costes que pudiese generar, en aras de alcanzar una mayor muestra y obtener datos novedosos y más reales (Lamas, 2010).

En 2005, la empresa IBOPE utilizó las señales de radio para transmitir esta información en tiempo real. Para este fin creó TC Net Real Time, un software capaz de medir la audiencia en directo, minuto a minuto. Dato que facilitó mucho a las cadenas la labor de programación de contenidos (MEDIA, 2015).

Aunque fueron cinco años los que tuvieron que pasar (de 2005 a 2010) para que IBOPE Paraguay, basándose en esta tecnología, presentase de manera oficial Real Time Rating, que permite la informatización de las cadenas de televisión y es capaz de mandar los datos a los usuarios que lo deseen a través de Internet (ABC, 2010).

Por muy rápida que sea la transmisión de datos, sigue siendo una medición tradicional, similar al audímetro; el único cambio relevante es la velocidad con la que viajan los datos y que pueden ser consultados por los usuarios de IBOPE Paraguay (Última Hora, 2010).

[12] En el mundo audiovisual se puede definir como la integración de diferentes realidades o medios, ya sean Internet, streaming, redes sociales, televisión lineal, etc. (Almeda, 2012). 
Con la llegada de las plataformas streaming no sólo ha evolucionado la manera de consumir contenido, sino la medición de esas audiencias. Al igual que ocurría con Nielsen, existe una empresa encargada de medir la audiencia de plataformas como Netflix, sin su consentimiento. Es el caso de Symphony, que mide los visionados que pueden alcanzar los contenidos de dicha plataforma, tanto de series actuales como de capítulos antiguos (MarketingDirecto, 2016).

Romesh Buchwalter, director de Symphony Technologic Group (la empresa encargada de Symphony) trabajó durante años en Nielsen hasta que fundó su propia empresa para dar el paso que las audiencias necesitaban (MarketingDirecto, 2016). Su finalidad era medir la eficacia de la publicidad crossmedia, pero en 2014 viraron su rumbo para calcular y entender la visión multiplataforma. La intención es que los usuarios utilicen una aplicación, que previamente se descargan en sus dispositivos móviles u ordenadores, para medir el contenido que consumen. La aplicación se llama VideoPulse, pero no se centra del todo en el contenido de la televisión lineal.

Actualmente, VideoPulse mide la programación de unos 210 canales en EE.UU., entre los que se encuentran Hulu o Netflix. Mediante un sistema de reconocimiento de audio, a través de los dispositivos de los usuarios, esta aplicación de Symphony cuenta ya con unos 15.000 panelistas, consiguiendo una muestra mayor a la ofrecida por la empresa encargada de la medición de audiencia de manera tradicional.

Uno de los inconvenientes de la aplicación se presenta cuando el usuario accede a contenidos en diferido, porque hay problemas para localizar dónde se está emitiendo, al contrario de lo que ocurre en un programa en directo. Por otro lado, la gran ventaja es que las cadenas de televisión no tendrían que invertir en nada, ni cambiar su infraestructura (Castro, 2015).

En España la empresa FLUZO, siguiendo la línea de Symphony, se encarga de ofrecer una medición de audiencia a través de una aplicación, mediante la captación de audio. Según Vicente García, cofundador de FLUZO, «ofrece una monitorización de televisión y radio 24 horas al día, los siete días de la semana, incluso teniendo en cuenta las campañas televisivas que se están emitiendo en ese momento concreto. Se desarrolló un sistema de adtracking ${ }^{13}$ que conecta al anunciante con su campaña para que puedan medir el éxito de su plan. Además, integra una aplicación móvil y se mide la audiencia de usuario-usuario por primera vez. Una herramienta que va a ser capaz de medir usuario-usuario en tiempo real» (García, 2018).

[13] Adtracking es un método de seguimiento publicitario en diferentes servidores para conocer el éxito, el fracaso o el alcance de la campaña publicitaria. 
Incluso Netflix ha comenzado a cambiar la manera de medir la audiencia de sus contenidos. En la televisión lineal muchos contenidos de calidad y con gran presupuesto se siguen jugando su continuidad con los datos ofrecidos por los audímetros ${ }^{14}$; en el caso de Netflix, los factores que determinan el éxito de sus series, películas o documentales se basan, principalmente, en el número de visualizaciones, la cantidad de espectadores que han consumido todos los capítulos de la serie, el triunfo en el primer mes de su publicación o la reputación de un determinado producto. No es necesario que los contenidos audiovisuales sean sentenciados por los datos que obtengan en una sola emisión, como ha ocurrido hasta ahora (Camacho, 2019).

Andrew Green, responsable de audiencias de la empresa Ipso, afirmó que en la actualidad se podía hablar de una medición 5.0, donde ya no son tan importantes los datos que se pueden obtener de una sola emisión, sino que la clave está en la unión de los diferentes datos que se puedan obtener usando distintas plataformas de visionado y de recogida de datos (Cortés, 2018).

En España se ha realizado por primera vez una medición en la que se incorporan los datos de diferentes fuentes, presentando un reporte con los datos Total Video; es decir, el visionado completo de un contenido televisivo en directo.

Este método se empleó con el programa Operación Triunfo 2018, siguiendo la estela del gran éxito cosechado en la edición anterior. Con el fin de conocer datos cuantitativos y cualitativos de los espectadores de este talent show musical, se utilizaron datos de la medición de audiencias de la televisión lineal (Kantar Media) y se añadieron los obtenidos por la web oficial de RTVE.es y el canal oficial del programa en YouTube (ComScore). Además de esto, The Cocktail Analysis ${ }^{15}$ realizó un estudio, junto con la colaboración de las empresas FLUZO y Netquest ${ }^{16}$, en el que utilizó una muestra de 1.800 espectadores, de entre 16 y 65 años, donde se midieron sus teléfonos móviles en relación con el consumo de este programa (Think With Google, 2019).

Este experimento ha permitido obtener de una manera clara y precisa toda la información referida al consumo de un programa de televisión; no solamente durante su emisión en directo, sino del comportamiento de los espectadores durante el transcurso del programa y en los días posteriores. De esta manera,

[14] Se puede poner de ejemplo el caso de la serie de televisión La Casa de Papel que se emitió, en primer lugar, en el prime time de Antena 3 sin obtener grandes datos. Meses después fue comprada por Netflix y se convirtió en la serie de habla no inglesa más vista del catálogo de la plataforma en streaming en 2018. Llegó a ganar un Emmy como mejor serie extranjera y se encargó una nueva temporada para la plataforma.

[15] Consultoría española de investigación de mercados.

[16] Empresa de servicios de encuestas online. 
se pudo conocer que, además de la audiencia que publicaba Kantar Media, después de la emisión en directo del programa, un 43 por ciento de los espectadores de Operación Triunfo revisionaban el contenido el día posterior a su emisión. Al mismo tiempo, un 72 por ciento de los espectadores de la emisión en directo continuaban, durante el transcurso de la semana, buscando y visionando contenidos VOD (video bajo demanda) relacionados con Operación Triunfo (Think With Google, 2019).

Este estudio ha permitido elaborar un análisis sobre los diferentes perfiles de los espectadores del programa, en el que se muestra que un 14 por ciento son digital puro; es decir, consumen todo el contenido a través de Internet, en diferentes dispositivos. Por otro lado, el perfil de espectador tradicional se corresponde con un 12 por ciento del total de los espectadores; un dato más que importante para la televisión lineal. Como se aprecia en la ilustración 2, el 10 por ciento del total de los espectadores de Operación Triunfo 2018 son consumidores intensivos multiplataforma - consumen todo el contenido que se ofrece del programa musical, en diversas plataformas-. También se comprobó que un 64 por ciento de los espectadores son esporádicos y consumían aproximadamente un total de siete minutos por contenidos ofrecidos en diferentes soportes, incluida la gala en directo (Think with Google, 2019).

\section{Ilustración 2. Resultados del estudio realizado sobre el consumo crossmedia de Operación Triunfo 2018.}

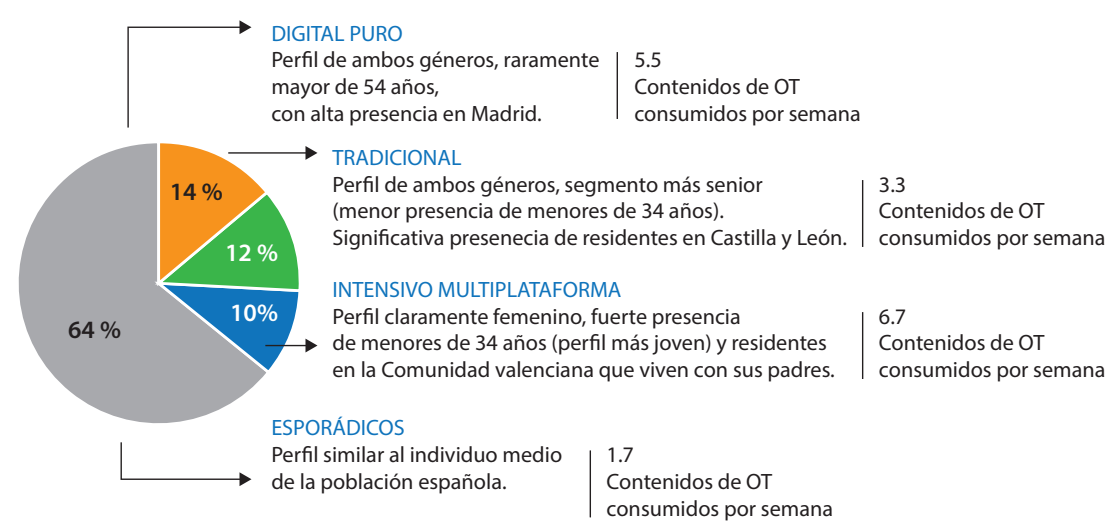

Fuente: Think With Google (2019).

Jennie Beck, jefa de operaciones de Kantar Media, señala que hay emisiones que aumentan su audiencia si se tiene en cuenta el consumo online. 
Además, espera que los datos que miden junto con ComScore puedan estar listos a lo largo de 2019, recordando que cadenas de televisión como TVE y TV3 ya disponen de ellos (Cortés, 2018).

Para Ricardo Vaca, presidente de Barlovento Comunicación, el camino del cambio no es el que se está dibujando. «Ni lo veo (el cambio) ni creo que se pueda dar, a no ser que los audímetros se modernicen, siempre dentro de un control por parte de Kantar Media, que es la responsable real de todo este entramado» (Vaca, 2017).

Desde el punto de vista de los anunciantes, Begoña Gómez, directora de la Asociación Española de Anunciantes comenta que «en estos momentos (la medición de audiencia tradicional) es la base que tenemos, pero debemos seguir evolucionando, ya no nos movemos buscando sólo datos demográficos del consumidor, sino que queremos conocer cuáles son sus hábitos comportamentales para llegar de la forma más adecuada. Es por ahí por donde la medición ha de moverse precisamente, para contactar con el consumidor de la mejor manera» (Gómez, 2018).

Independientemente de las diferentes opiniones que puedan existir al respecto, hay varias opciones que suenan con fuerza cuando se habla de la medición de la televisión lineal en un futuro próximo. Una de ellas es la medición de audiencias híbrida.

\section{Medición híbrida}

Todas las mañanas se publican las audiencias de los contenidos emitidos en la televisión tradicional durante el día anterior. Estos datos son esperados por muchos ya que, según lo publicado, un determinado programa puede fracasar o triunfar.

La información obtenida, con los datos ofrecidos por Kantar Media, es la que provoca que una cadena retire un producto, lo reprograme e incluso sirva para medir el éxito de todo el canal. Estos datos, además, favorecen la repartición entre los diferentes grupos audiovisuales de la inversión publicitaria en televisión. En 2018 esta inversión sufrió un estancamiento. Por segundo año consecutivo se estiman unas cifras de más de 2.100 millones de euros, lo que supone casi un dos por ciento del Producto Interior Bruto español (Europa Press, 2019).

Vicente García, cofundador de FLUZO, utiliza un símil musical para comprender el proceso de digitalización: «Al principio la industria no quería Spotify y, al final, la sociedad ha hecho que todas las discográficas quieran estar en Spotify. Lo mismo ocurre con la industria editorial, los grandes dinosaurios de las discográficas se han podido salvar, pero ¿y los que vienen nuevos?». 
Señala al mismo tiempo la importancia de analizar el mercado actual y no olvidarse de que el mundo audiovisual también puede cambiar de manera drástica. «Hay quién dice que Netflix no es competidor porque no tiene publicidad, pero sí te quita espectadores. Es decir, si surgen nuevos formatos que están quitando clientes potenciales al sector. Se está consumiendo mucho contenido audiovisual, más que nunca me atrevería a decir. La gente ve el contenido de otra manera» (García, 2018).

Es preciso estudiar no sólo cómo aprovechar el nacimiento de estas nuevas plataformas de contenido on demand, como ocurrió con el citado caso de La casa de papel, sino también de qué manera se pueden satisfacer los deseos de las cadenas y de los anunciantes en este nuevo panorama.

El consumo de vídeo on demand (VOD) y en diferido empieza a tenerse en cuenta para lograr una imagen completa de las personas que ven un contenido determinado fuera de la emisión original. No existe un estándar europeo de medición de audiencia de contenidos en diferido y esta medición es importante para muchos de ellos, sobre todo series y otros eventos que no se producen en directo (Quintas y Neira, 2018).

El nuevo comportamiento del usuario, que es el consumo multipantalla o multitask, a su vez también da lugar a lo que se conoce como multiplataforma: la distribución de un contenido por varios dispositivos (Diego, 2015). Nace así lo que se acuña como Cross Media Audience Measurement o, lo que es lo mismo, la medición integral de la audiencia multiplataforma, que hoy en día es uno de los grandes retos del sector (Quintas y Neira, 2018).

Los datos que se llegarían a obtener con este tipo de medición son beneficiosos para todo el sector; no sólo para productoras, cadenas o espectadores, sino también para los anunciantes que, al fin y al cabo, son los que permiten que este sector siga en funcionamiento con su inversión constante. De hecho, una de las consecuencias de este tipo de medición es la de ofrecer a los anunciantes GRP'S (Gross Rating Points) ${ }^{17}$ y los impactos, la frecuencia y la cobertura de una campaña determinada tanto online como offline.

Alejandro Perales Albert, asesor técnico de la Asociación de usuarios de la comunicación, afirmaba, a la hora de hablar de una nueva medición de audiencia, que «el modelo ha ido introduciendo mejoras, como las ampliaciones muestrales, el cómputo de invitados, el consumo en diferido y los segundos televisores. Hay que avanzar en ese camino, y, sobre todo (...), ir hacia un modelo global que permita medir el visionado ya sea lineal o no lineal, en

[17] El número total de impactos que una campaña puede ejercer por cada cien personas de su público objetivo dentro del conjunto de espectadores. También se puede explicar como la presión publicitaria de una sola inserción (Patel, 2016). 
cualquier pantalla, y evaluar adecuadamente el impacto global de las comunicaciones comerciales» (Perales, 2018).

Por su parte, Ignacio Gómez, director de Análisis y Nuevos Proyectos de RTVE, sólo ve posible un cambio si hay «una unión muy grande, repartir los datos de manera correcta, dividir mejor el mercado, etc.» (Hernández, 2018). Y ahí se encontraría el núcleo principal de esta investigación: crear una medición híbrida total que sea capaz de aportar datos cuantitativos y cualitativos de una calidad óptima y así anticiparse al posible cambio que pueda tener lugar en un corto espacio de tiempo dentro del sector audiovisual.

\section{La medición híbrida total}

Alejandro José Tapia Frade, profesor adjunto de la Universidad Loyola de Sevilla, recuerda que es necesario no olvidar que el panel usado en la medición de audiencias en España es mucho más que correcto. De hecho, es el séptimo mejor del mundo, detrás de países como Estados Unidos o China y el cuarto mejor medidor de audiencia de Europa, precedido por Francia, Rusia y Austria. El problema, según Tapia Frade, no es el panel sino el método de medición (Tapia, 2019).

El modelo que aquí se plantea no pretende cambiar el trabajo ni los datos aportados por Kantar Media, sino mostrar la necesidad de involucrar a una serie de actores - y la información que son capaces de obtener- para que, tras una combinación de todos ellos se puedan obtener datos que mejoren, amplíen y ofrezcan una información cuantitativa y cualitativa del espectador que pueda ser beneficiosa para los anunciantes, para las cadenas, las productoras y los propios espectadores de televisión.

Existen varios pasos para conseguir una medición crossmedia, una medición multimedia o una medición que tenga en cuenta las emisiones online y offline de un contenido determinado. Eduard Nafría, director de Insight y Desarrollo de Negocio de Kantar Media, afirmó durante el congreso $\mathrm{AEDEMO}^{18}$ celebrado en febrero de 2019 que, «a pesar del descenso de consumo de la televisión en España, el 42 por ciento de la población consume más ahora que antes. Hay que mantener una televisión tradicional, pero es más importante que nunca incorporar una medición transdigital debido al consumo multidispositivo» (Nafría, 2019).

Para Nafría, este tipo de medición es avanzar en su acuerdo con ComScore que, desde 2015 hasta febrero de 2019, cuando fueron públicos los resultados del estudio que se elaboró para Operación Triunfo 2018, no ha podido avanzar lo suficiente. Y aquí es donde reside una de las principales claves para 
entender cómo debe ser esta medición híbrida total; una que incluya en estos estudios a muchos más actores, para que los datos que se obtengan sean lo más completos posibles.

A la hora de pensar en un modelo de medición, teniendo en cuenta la situación actual, parece inviable que se puedan colocar dispositivos o softwares con funciones similares a las del audímetro de Kantar Media en cada uno de los dispositivos que se usan para el consumo de contenido audiovisual. A continuación, se presentan los actores que deberían estar involucrados en la medición de audiencia híbrida total que aquí se propone:

$\rightarrow$ Kantar Media

$\rightarrow$ ComScore

$\rightarrow$ Proveedores de televisión por suscripción

$\rightarrow \mathrm{HbbTV}^{19}$

La mayoría de actores involucrados en la medición híbrida total aportarían una gran información cuantitativa, por lo que es fundamental contar con aquellos actores capaces de ofrecer datos cualitativos. Es posible que los decodificadores de los proveedores de televisión por suscripción o los propios sistemas de HbbTV con LOVEStv ofrezcan información cualitativa también, pero para ello es necesario que hagan partícipe al usuario, ya no sólo para que

[19] Hybrid Broadcast Broadband Tv (HbbTV) es un estándar europeo que permite crear un sistema que posibilita a los canales de televisión añadir una capa de información digital en los programas de televisión tradicional (Mira, 2018). RTVE, gracias a la HbbTV, presentó un sistema que permite, a través de un televisor conectado a Internet, requisito básico para el funcionamiento del HbbTV, consultar noticias, recibir información y visionado de cualquier contenido de RTVE a la carta gracias a este sistema (Villa, 2018). Al mismo tiempo, Mediaset España, Atresmedia y RTVE, demostrando el buen momento propiciado por la evolución tecnológica y la conexión que existe en los hogares en España, presentan una plataforma HbbTV llamada LOVEStv, que llega a un público potencial de dos millones de personas (Barlovento, 2019), creando así un valor añadido para los espectadores de la televisión lineal. Este sistema permite, mediante una SmartTV, consumir los contenidos emitidos por las cadenas con una semana de antigüedad, comenzar desde el inicio programas cuya emisión ya esté en curso y una guía de programación mejorada (Bluper, 2015). Lo más interesante del HbbTV, a la hora de hablar de medición de audiencia total, es que ofrece a las cadenas que lo usan una información cuantitativa en tiempo real sobre los espectadores que están consumiendo un contenido determinado. Aunque, por otro lado, la segmentación no está bien realizada y sería un aspecto que se tendría que mejorar. Además, Ignacio Gómez señala que una de las claves serían los fabricantes de televisiones inteligentes, ya que son los creadores de estas televisiones que permiten las mediciones mencionadas (Gómez, 2018). 
esté conforme con la utilización de sus datos, sino para que participen en su obtención.

Se ha presentado un escenario similar al actual, con la unión de Kantar Media y ComScore, con la posibilidad de ir más allá, haciendo uso de las oportunidades que ofrece el panorama actual. La colaboración entre competencias, impensable hasta hace escasos meses, señala un camino lleno de alianzas que favorezcan el mercado audiovisual.

La última noticia que abre la puerta a un verdadero cambio en la medición de audiencia de la televisión lineal en España viene de la mano del grupo Atresmedia con la creación de VAR (Video Advertising Reach). Esta nueva herramienta, que será presentada de manera oficial en diciembre de 2019 y de la que aún se desconoce cómo será su funcionamiento, aúna los datos de sus contenidos emitidos en televisión y en Internet para ofrecer a los anunciantes más detalles sobre el impacto real de sus campañas en las cadenas del grupo televisivo. Aún con mucho hermetismo alrededor de la noticia, Atresmedia promete que esta herramienta será sólo la punta del iceberg (El confidencial, 2019).

\section{Conclusiones}

El nuevo paisaje señala cuál debería ser el camino a seguir para construir una medición de audiencias lo más completa posible, que exprima cada posibilidad, con el fin de obtener un incremento de la inversión publicitaria y, al mismo tiempo, ofrecer datos que faciliten la creación de una publicidad personalizada para los espectadores. La cantidad de datos de usuario que se manejarían con un sistema de medición como el que se ha planteado a lo largo de este artículo se aproximaría a la ciencia del Big Data ${ }^{20}$.

Para crear un sistema de medición de este calibre se debe trabajar siempre dentro del reglamento europeo. Uno de los primeros pasos, y casi de mayor relevancia, es tener en cuenta al espectador. Sólo haciéndoles partícipes a los usuarios, ofreciéndoles información del nuevo proceso, se podrá servir una mejor experiencia publicitaria y audiovisual, necesaria para salvar el modelo televisivo en nuestro país.

Por otra parte, es importante la creación de una arquitectura de datos unificada, que sea empleada por cada uno de los actores involucrados en la medición híbrida total (PowerData, 2016). Para ello, sería fundamental la existencia de un organismo público, que luche por el sector y por la continuidad y mejora de la inversión publicitaria. Que se encargue de trabajar con los

[20] Se podría definir como un conjunto de datos o combinaciones de conjuntos de datos con un volumen, velocidad y complejidad tal que no pueden ser tratados mediante recursos tradicionales (Ardid e Irene, 2017). 
datos que cada actor involucrado ofrezca, pero que no trabaje en la medición o recogida de los mismos. Así, existiría una garantía y fiabilidad total de los resultados de una medición tan compleja como la medición híbrida total, cuyos datos podrían ofrecer una de las informaciones más exactas, completas y segmentadas de la industria de la medición de audiencias.

Además, aprovechando lo que aquí se explica, se cumpliría la Ley 7/2010, del 31 de marzo, General de La Comunicación Audiovisual, donde se propone la creación de un Consejo Estatal de Medios Audiovisuales. Este instrumento sería perfecto para la supervisión, recopilación y publicación de los datos que recogen los diferentes actores involucrados en la medición de audiencia de la televisión lineal.

A continuación, se presentan los puntos concluyentes, teniendo en cuenta los objetivos planteados, que reflejan una respuesta global del estudio que aquí se ha desarrollado.

$\rightarrow$ El nuevo panorama audiovisual ha dado pie a mejoras y modificaciones interesantes. La medición crossmedia fue una de las que más expectación causó en 2015, cuando Kantar Media y ComScore se unieron para mejorar la medición actual y dar un paso más allá dentro del sector audiovisual, al incluir también los consumos digitales. Este gran paso concluyó casi cuatro años después, tras presentar los resultados de los programas estudiados, además de tener en cuenta su emisión en directo, así como sus visionados bajo demanda (VOD) u online.

$\rightarrow$ Podemos hablar de televisión fluida o líquida, donde los contenidos fluyen por diferentes canales para facilitar la elección de los espectadores. La medición crossmedia o transmedia fusiona los datos obtenidos por los audímetros de Kantar Media y los datos de ComScore para ofrecer una información mucho más completa, aunque por el momento es necesario atraer a otros actores para conseguir un escenario mucho más perfecto y elaborado.

$\rightarrow$ De esta manera, surge la Medición de audiencia híbrida total, con un gran número de actores involucrados, cada uno especializado en un sector concreto. Esta nueva medición aportaría infinidad de oportunidades de trabajo Big Data. La ingesta de estos datos obligaría a cambiar el workflow actual de las empresas de análisis de audiencias, para garantizar una fiabilidad y calidad de los datos obtenidos. Al 
mismo tiempo, también obligaría a asegurar el derecho a la protección de datos de los usuarios, amparados por el Reglamento Europeo ${ }^{21}$. Al mismo tiempo, se puede culpar por la tardanza en la creación de un nuevo modelo de medición a la falta de recursos económicos destinados a este fin. El sistema actual, tal y como está, beneficia a los dos grandes grupos de televisión, Atresmedia y Mediaset.

$\rightarrow$ Se plantea la existencia de un organismo público que ejerza de árbitro para defender la continuidad y el buen funcionamiento del sector $\mathrm{y}$, al mismo tiempo, garantizar la inversión publicitaria, tan importante para el sector audiovisual. Este organismo se encargaría de los análisis y auditorías necesarios para asegurar la calidad y la veracidad de los datos de la audiencia híbrida total, siempre dejando libertad a los actores involucrados en la misma. De esta manera, se aplicaría la Ley 7/2010, del 31 de marzo, General de La Comunicación Audiovisual, donde se aboga por la creación de un Consejo Estatal de Medios Audiovisuales, órgano que podría, sin ningún problema, acoger el cometido que aquí se expone.

Para concluir, se puede afirmar que aún es pronto para establecer un sistema de medición de audiencia para la televisión lineal que abarque los diferentes tipos de visionado de contenidos a los que un espectador puede acceder. La revolución digital cambia y fluye de una forma rápida, mucho más de lo que se pueda imaginar. De esta manera, el sector es muy sensible a los cambios que tienen lugar. Un ejemplo de ello es el rápido crecimiento de los sistemas streaming o la muerte del DVD o el Blu-Ray en apenas cinco años.

De ahí la gran importancia de prever cómo va a evolucionar el sector audiovisual, e ir un paso por delante. Es necesario no olvidar que los grandes protagonistas de la medición de audiencia son los anunciantes ya que, gracias a su inversión, el sector audiovisual sigue adelante. Los sistemas de medición que existen o que existan en un futuro próximo deberán ofrecer una información que satisfaga a los anunciantes y que sea lo más real posible, incluyendo no sólo la televisión lineal, sino los diferentes modelos de consumo, para ofrecer una visión completa de cómo está cambiado el mundo audiovisual.

Debido a la protección que necesitan los espectadores y usuarios audiovisuales, y lo que supone la inversión publicitaria al PIB de este país, sería de gran

[21] Reglamento Europeo (UE) 2016/679 del Parlamento europeo y del consejo del 27 de abril de 2016 relativo a la protección de datos personales y a la libre circulación de estos datos y por el que se deroga la Directiva 95/46 CE (Reglamento general de protección de datos). 
importancia la existencia de un organismo público imparcial, que se encargue de velar por los usuarios, la calidad de los datos que se ofrezcan y sirva de ayuda a las diferentes empresas encargadas de la medición de audiencias.

\section{Bibliografía}

$A B C$ (2010). Real Time Rating de Ibope Paraguay. $A B C, 10$ de junio. En https://www.abc.com.py/edicion-impresa/sociales/real-time-rating-de-ibope-paraguay-114913.htm

Ardid, B. (2017). Synergic Academy diseña programas formativos en Big Data bonificables para las empresas. RRHH Digital, 17 de octubre.

Barlovento (2018). Análisis de audiencias de televisión: octubre 2018. Madrid: Barlovento Comunicación.

Barlovento (2019). Análisis Televisivo 2018. Madrid: Barlovento Comunicación. BArredo, A. (2017). Cada vez menos hogares en España sin televisión por culpa del Smartphone. La Vanguardia, 1 de agosto. Recuperado desde http://www. lavanguardia.com/tecnologia/20170801/43264277786/televisores-espana-smartphones-ocio.html

BArrio, J. M. (2012). El tuitómetro cuestiona la eficacia del audímetro. El País, 19 de diciembre. En https://elpais.com/tecnologia/2012/12/18/actualidad/1355829956_973495.htm Bluper (2015). ¿Qué es la tecnología HbbTV? ¿Para qué va a servir el botón azul de nuestra tele? El Español, 25 de mayo. Recuperado desde https://www. elespanol.com/bluper/noticias/que-es-la-tecnologia-hbbtv-boton-azul-lovestv

Camacho, J. G. (2019). Las series ya no se juegan su éxito en un solo día como pasaba con las emisiones lineales de la TV tradicional. Internet ha abierto las puertas del campo. El Comercio, 3 de febrero.

Castro, A. (2015). VideoPulse, la nueva herramienta que mide audiencias en TV. Mobile Outdoor Magazine, 15 de septiembre. Recuperado desde http:l/ www.mobileoutdoormagazine.com/tecnosfera/videopulse-la-nueva-herramienta-que-mide-audiencias-en-tv

Claes, F. y Dettell, L. (2015). Audiencia Social en Twitter: hacia un nuevo modelo de consumo televisivo. Fonaments, 36, 111-132.

Cortés, H. (2018 ). Así se medirán las audiencias en el futuro. $A B C, 5$ de junio.

Diego, P. G. (2015). Estrategia multiplataforma de la televisión conectada. Editorial Fraga, 145-168.

Dircomfidencial (2016). La revolución de audiencias promovida por Kantar Media y ComScore se retrasa. Dircomfidencial, 13 de junio. Recuperado desde https://dircomfidencial.com/marketing/la-revolucion-de-audiencias-promovida-por-kantarmedia-y-comscore-se-retrasa-20160613-0404/

El Confidencial (2019). Atresmedia crea una herramienta para sumar la audiencia de televisión y de Internet. El Confidencial, 4 de octubre de 2019. En https:/l www.elconfidencialdigital.com/articulo/medios/atresmedia-crea-herramienta-sumar-audiencia-television-internet/20191003194952131908.html 
EuropaPress (2019). La inversión de publicidad en televisión se estanca por segundo año consecutivo, según Barlovento. EuropaPress, 1 de enero. En https://www.europapress.es/sociedad/noticia-inversion-publicidad-television-estanca-segundo-anoconsecutivo-barlovento-20190102174138.htm|

Frade, A. J. (enero de 2019). La medición de audiencias de la televisión lineal (entrevista personal no publicada de J. A. Quesada).

Freire, F. C. (2015). Adaptación de los Medios Tradicionales a la Innovación. StudyLib, 4, 445.

García, V. (25 de Julio de 2018). La medición de audiencias de la televisión lineal (entrevista personal no publicada de J. A. Quesada).

Gómez, B. (noviembre de 2018). La medición de audiencias de la televisión lineal (entrevista personal no publicada de J. A. Quesada).

HERnÁNDEZ, I. G. (19 de marzo de 2018). La medición de audiencias de la televisión lineal (entrevista personal no publicada de J. A. Quesada).

Hidalgo, E. S. (2018). Los audímetros no son infalibles: por qué un $0 \%$ de 'share' no significa cero espectadores. El País, 7 de enero.

Kantar Ibope Media (2015). Medición de Audiencia de TV (abierta y paga). Kantar Media. Recuperado desde http://www.kantaribopemedia.com.arlibope/wp/television

Keltie, E. (2017). The Culture Industry and Participatory Audiences. Palgrave MacMillan.

La Vanguardia (2017). ¿Por qué las audiencias no volverán a ser lo mismo? La Vanguardia, 22 de febrero.

Lamas, C. (2010). El uso de la tecnología en la medición de las audiencias. AIMC: Asociación para la Investigación de Medios de Comunicación, 6 de octubre.

Madinaveitia, E. y Marchante, M. (2015). Medición de audiencias: desafío y complejidad en el entorno digital. Harvard Deusto marketing y ventas, 131, 26-33.

Marín, F. (2018). Netflix llega a Movistar: cómo funciona el servicio y cuáles son los nuevos precios. $A B C, 11$ de diciembre.

Marinelli, A. y Celata, G. (2012). Connecting Televisión. Italia: Guerini e associati.

Marinelli, A. y Celata, G. (2014). La Televisione Dopo la Televisione. Roma: Sapienza Universitat di Roma.

MarketingDirecto (2016). Así funciona la innovadora empresa que ha medido las audiencias de Netflix sin su aprobación. MarketingDirecto, 21 de enero. Recuperado desde https://www.marketingdirecto.com/anunciantes-general/medios/asi-funcioha-la-innovadora-empresa-ha-medido-las-audiencias-netflix-sin-aprobacion 
MirA, A. (2018). HBBTV, qué es esta tecnología y cómo activarla en tu tele. Tu Experto, 15 de junio. Recuperado desde https:/www.tuexperto.com/2018/06/15/hbbtvesta-tecnologia-activarla-tele/

NovoA, J. (2014). El Futuro de la SocialTV. Genbeta. Obtenido de https://www. genbeta.com/web/el-futuro-de-la-socialtv-es-apasionante-carlos-sanchez-cofundador-de-thedata-republic-tuitele

PATEL, S. (2016). WTF is a GRP? Digiday, 17 de noviembre. Recuperado desde https://digiday.com/marketing/what-is-a-grp-gross-ratings-point/

Perales Albert, A. (noviembre de 2018). La medición de audiencias de la televisión lineal (entrevista personal no publicada de J. A. Quesada).

PowerData (2016). Big Data: ¿En qué consiste? Su importancia, desafíos y gobernabilidad. Power Data. Recuperado desde https://www.powerdata.es/big-data

Prompy, D. P.; Du Toit, H. D.; Griffin, H. J.; TajadurA-Jimenez, A. y Cox, A. L. (2014). Working with the television ON: An investigation into media multitasking. University College London.

Puromarketing (2014). Kantar Media lanza el primer sistema oficial de medición de Twitter. Puromarketing, 4 de diciembre. Recuperado desde https:/l www.puromarketing.com/16/23570/kantar-media-lanza-primer-sistema-oficial-medicion-twitter.html

PuroMarketing (2018). El Audíemtro como medidor de audiencias televisivas es ya un sistema obsoleto. PuroMarketing, 26 de diciembre. Recuperado de https://www.puromarketing.com/45/31432/audimetro-como-medidor-audiencias-televisivas-sistemaobsoleto.html

Quintas, N. y González Neira, A. (2014). Audiencias Activas: participación de la audiencia social en la televisión. Comunicar, 43, 86-90.

Quintas Froufe, N. y GonzÁlez NeIra, A. (coord.) (2015). La participación de la audiencia en la televisión: de la audiencia activa a la social. Madrid: AIMC. Recuperado de https://www.aimc.es/a1mc-c0nt3nt/uploads/2017/05/2016_01participacion_audiencia_tv.pdf

Quintas, N. y González NeIra, A. (2018). Consumo Televisivo y su medición en España: camino hacia las tendencias híbridas. El Profesional de la Información, 3, 380-382.

REGIDOR, R. (2013). Ya se puede medir el 'share social' en los programas de televisión. Libre Mercado, 9 de enero. Recuperado desde https://www.libremercado. com/2013-09-01/ya-se-puede-medir-el-share-social-en-los-programas-de-television-1276498213/

Rodrigo, B. (2018). El imparable negocio de la publicidad en televisión. $A B C$, 24 de noviembre.

Santiago, F. y GónZalez, I. (2015). Nuevos tiempos y nuevos usos del tiempo libre en torno al consumo audiovisual. $31^{\circ}$ Seminario de televisión Aedemo. 
Think With Google (febrero de 2019). Operación Triunfo: cuando televisión y digital se unen para conseguir el éxito. Think with Google. Recuperado de https://www.thinkwithgoogle.com/int/les-es/canales-de-publicidad/video/operacion-triunfo-cuandotelevision-y-digital-se-unen-para-conseguir-el-exitol

UltimaHora (2010). Ibope revolucionará con medición en tiempo real. ÚltimaHora, 11 de junio. Recuperado de https://www.ultimahora.com/ibope-revolucionara-medicion-tiempo-real-n329727.html

VACA, R. (enero de 2017). La medición de audiencias de la televisión lineal (entrevista personal no publicada de J. A. Quesada).

Vertele (2019). Las 10 emisiones más vistas de la temporada. Madrid: Vertele. Recuperado de http://vertele.eldiario.es/audiencias-canales/analisis-espana/audiencias-analisisespecial-emisiones-mas-vistas-temporada-2018-eurovision-amaia-alfred_0_1991800808.htm|

Para citar este artículo: Cortés Quesada, J. A. y Barceló Ugarte, T. (2019). La medición de televisión en España: estado del arte y propuesta para el cálculo de la audiencia híbrida total. index.comunicación, 9(1), 217-242. 